\title{
Large Scale Simulations of Astrophysical Jets
}

\author{
J. H. Beall* \\ St. John's College, USA \\ E-mail: beallesjc.edu \\ D.V. Rose, and Kevin Lind
}

Voss Scientific, Albuquerque, NM, USA

Michael T. Wolff

Space Sciences Division, Naval Research Laboratory, Washington, DC, USA

Brian van Soelen, Izak van der Westhuizen, and Pieter Meintjes

University of the Free State, Bloemfontein, SA

\begin{abstract}
We present recent results of our three-dimensional (3-D) simulations of astrophysical jets. These efforts use a highly parallelized version of the $\mathrm{VH}-1$ Hydrodynamics Code in the hydrodynamics (HD) regime (Collella and Woodward 1984, and Saxton et al. 2005 in the hydrodynamic regime; and the PLUTO code (Mignone et al. 2007) for the hydrodynamic, magneto-hydrodynamic (MHD), relativistic hydrodynamic (RHD), and relativistic, magnetohydrodynamic (RMHD) simulations. We also continue our investigation using particle-in-cell simulations to benchmark a wave-population model of the two-stream instability and associated plasma instabilities in order to determine the energy deposition and momentum transfer rates for these modes of the jetambient medium interactions. In this regard, we believe that it is important to note that "simple" HD, RHD, and RMHD simulations are unable to show the real effects of very small scale (at least in terms of the scales of the simulations) plasma processes. Thus, these effects are being considered for use in a multi-scale code that incorporates energy deposition rate and momentum transfer from strong plasma turbulence generated by the interaction of the astrophysical jet with the ambient medium through which it propagates (Beall, 2010). In this work, we show some results from the modeling of these jets for a fully 3-D simulation of relativistic jets using the PLUTO code in the RHD regime. The jets in question have a relativistic velocity $\beta=0.80 c$. We show a specific example where the disk gravitational potential affects the jet propagation.
\end{abstract}

Keywords: jets, active galaxies, blazars, intracluster medium, non-linear dynamics, plasma astrophysics, computational fluid dynamics

Frontier Research in Astrophysics - II

23-28 May 2016

Mondello (Palermo), Italy

${ }^{*}$ Speaker. 


\section{Introduction}

Recent, high-resolution (VLBA) observations of astrophysical jets (see, e.g., Lister et al. 2009) and Beall (2015) for reviews) reveal complex structures in the cores of AGNs and microquasars apparently caused by ejecta from the central engine. The temporal and spatial structures of the jets at both microquasar and quasar scales (i.e., from milliparsecs to kiloparsecs), suggest that the effects might be produced by quasi-periodic processes in accretion disks. The ejected material then interacts with the surrounding interstellar material in the source region, that is, the BroadLine Region (BLR) and Narrow-Line Region (NLR) clouds, and with ejecta from prior episodes of activity. A particularly interesting example of these complex interactions is shown by the galactic microquasar, Sco X-1 (Fomalhaut, Geldzahler, and Bradshaw, 2001).

Such observations can be used to inform models of the jet-ambient-medium interactions. Based on an analysis of these data, we posit that a significant part of the observed phenomena come from the interaction of the ejecta with prior ejecta as well as interstellar material.

\section{Scales of Jet Interactions with the Ambient Medium}

Large-scale hydrodynamic simulations can be used to illuminate a number of interesting consequences of the jet's interaction with the ambient medium through which it propagates. These include acceleration and entrainment of the ambient medium, the effects of shock structures on star formation rates, and other effects originating from ram pressure and turbulence generated by the jet (see, e.g., Basson and Alexander, 2002; Zanni et al. 2005; and Krause and Camenzind 2003; Perucho 2012).

We believe, however, that hydrodynamic (HD), magneto-hydrodynamic (MHD) and (RMHD) simulations neglect important aspects of physics: the microscopic interactions that occur because of the effects of particle-particle interactions and the interactions of particles with the collective effects that accompany a fully or partially ionized ambient medium (i.e., a plasma). This is a similar problem to that presented by the estimation of viscosity in hydrodynamic simulations.

Though the physical processes (including plasma processes) in the ambient medium can be modeled in small regions by Particle-in-Cell (PIC) codes for some parameter ranges, simulations of the larger astrophysical jet structure with such PIC codes are not possible with current or foreseeable computer systems. For this reason, we have modeled plasma processes in the astrophysical regime by means of a system of coupled differential equations which give the wave populations generated by the interaction of the astrophysical jet with the ambient medium through which it propagates. A detailed discussion of these efforts can be found, variously, in Scott et al. (1980), Rose et al. (1984), Rose et al. (1987), Beall (1990), and Beall et al. (2003). The scales of these interactions is small compared to most hydrodynamic simulations, and their time scales are typically very fast compared to the overall evolution time of the hydrodynamical regime.

\subsection{Energy Loss, Energy Deposition Rate, and Momentum Transfer from Plasma Processes}

The system of equations used to determine the normalized wave energy densities is very stiff. Scott et al. (1980) estimated the equilibrium solution of this system of equations for heating of clusters of galaxies, and Rose et al. (1984) and Beall (1990) showed dynamical solutions that 
confirmed the stability of the equilibrium solutions. Solving the system of equations yields a timedependent set of normalized wave energies (i.e., the ratio of the wave energy divided by the thermal energy of the plasma) that are generated as a result of jets interaction with the ambient medium. These solutions can yield an energy deposition rate $(d E / d t)$, an energy deposition length $(d E / d x)$, and ultimately, a momentum transfer rate $(d p / d t) \sim\left(1 / v_{b}\right) *(d E / d t)$, where $v_{b}$ is the beam velocity. This $d p / d t$ can be used to estimate the effects of plasma processes on the hydrodynamic evolution of the jet.

For this part of the analysis, we suppose that a portion of the jet is composed of relativistic particles of either $e^{ \pm}, p-e^{-}$, or more generally, a charge-neutral, hadron- $e^{-}$jet, with a significantly lower density than the ambient medium. The primary energy loss mechanism for the electronpositron jet is via plasma processes, as Beall (1990) notes. Kundt $(1987,1999)$ also discusses the propagation of electron-positron jets.

Beall et al. (2006) illustrate two possible solutions for the system of coupled differential equations that model this mode of the jet-ambient medium interaction: a damped oscillatory and an oscillatory solution. The Landau damping rate for the two-temperature thermal distribution of the ambient medium is used for these solutions.

The average energy deposition rate, $\left\langle d\left(\alpha \varepsilon_{1}\right) / d t>\right.$, of the jet energy into the ambient medium via plasma processes can be calculated as $\left\langle d\left(\alpha \varepsilon_{1}\right) / d t>=n_{p} k T<W>\left(\Gamma_{1} / \omega_{p}\right) \omega_{p} \quad \mathrm{ergs}-\mathrm{cm}^{-3} \mathrm{~s}^{-1}\right.$, where $k$ is Boltzmann's constant, $T$ is the plasma temperature, $\langle W\rangle$ is the average (or equilibrium) normalized wave energy density obtained from the wave population code, $\Gamma_{1}$ is the initial growth rate of the two-stream instability, and $\alpha$ is a factor that corrects for the simultaneous transfer of resonant wave energy into nonresonant and ion-acoustic waves. The energy loss scale length, $d E_{\text {plasma }} / d x=-\left(1 / n_{b} v_{b}\right)\left(d \alpha \varepsilon_{1} / d t\right)$, can be obtained by determining the change in $\gamma$ of a factor of 2 with the integration $\int d \gamma=-\int\left[d\left(\alpha \varepsilon_{1}\right) / d t\right] d l /\left(v_{b} n_{b} m^{\prime} c^{2}\right)$ as shown in Rose et al. (1978) and Beall (1990), where $m^{\prime}$ is the mass of the beam particle. Thus,

$$
L_{p}(\mathrm{~cm})=\left((1 / 2) \gamma c n_{b} m c^{2}\right) /\left(d \alpha \varepsilon_{1} / d t\right)
$$

is the characteristic propagation length for collisionless losses for an electron or electronpositron jet, where $d \alpha \varepsilon_{1} / d t$ is the normalized energy deposition rate (in units of thermal energy) from the plasma waves into the ambient plasma. In many astrophysical cases, this is the dominant energy loss mechanism (Rose et al. 1984, Beall 1990). We can therefore model the energy deposition rate $(d E / d t)$, the energy loss per unit length $(d E / d x)$, and ultimately the momentum loss per unit length $(d p / d x)$ due to plasma processes, and can add these as an external force term in the PLUTO code.

Beall et al.(2015) show a late-time appearance of a fully three-dimensional simulation of an astrophysical jet gas density structure seen in an X-Z cross-section with a bulk velocity of $v=0.80 c$. In that work, they showed a simulation with $1024^{3}$ grid cells The simulation demonstrated welldeveloped Rayleigh-Taylor instabilities at the jet-ambient medium boundary. The simulation size presented was $\sim 5 \mathrm{kpc}$ on a side and showed the structures developed after $2 \times 10^{3}$ time steps (roughly $10^{4}$ years). In that simulation, the jet-to-ambient medium density ratio is $1 / 100$ and the ratio of jet input pressure to ambient medium pressure is $5 \times 10^{-3}$

Rose et al. (2002), and Beall et al.(2010) have compared the results of a PIC code simulations of an electron-positron jet propagating through an ambient medium of an electron-proton plasma with the solutions obtained by the wave population model code, and have found good agreement 


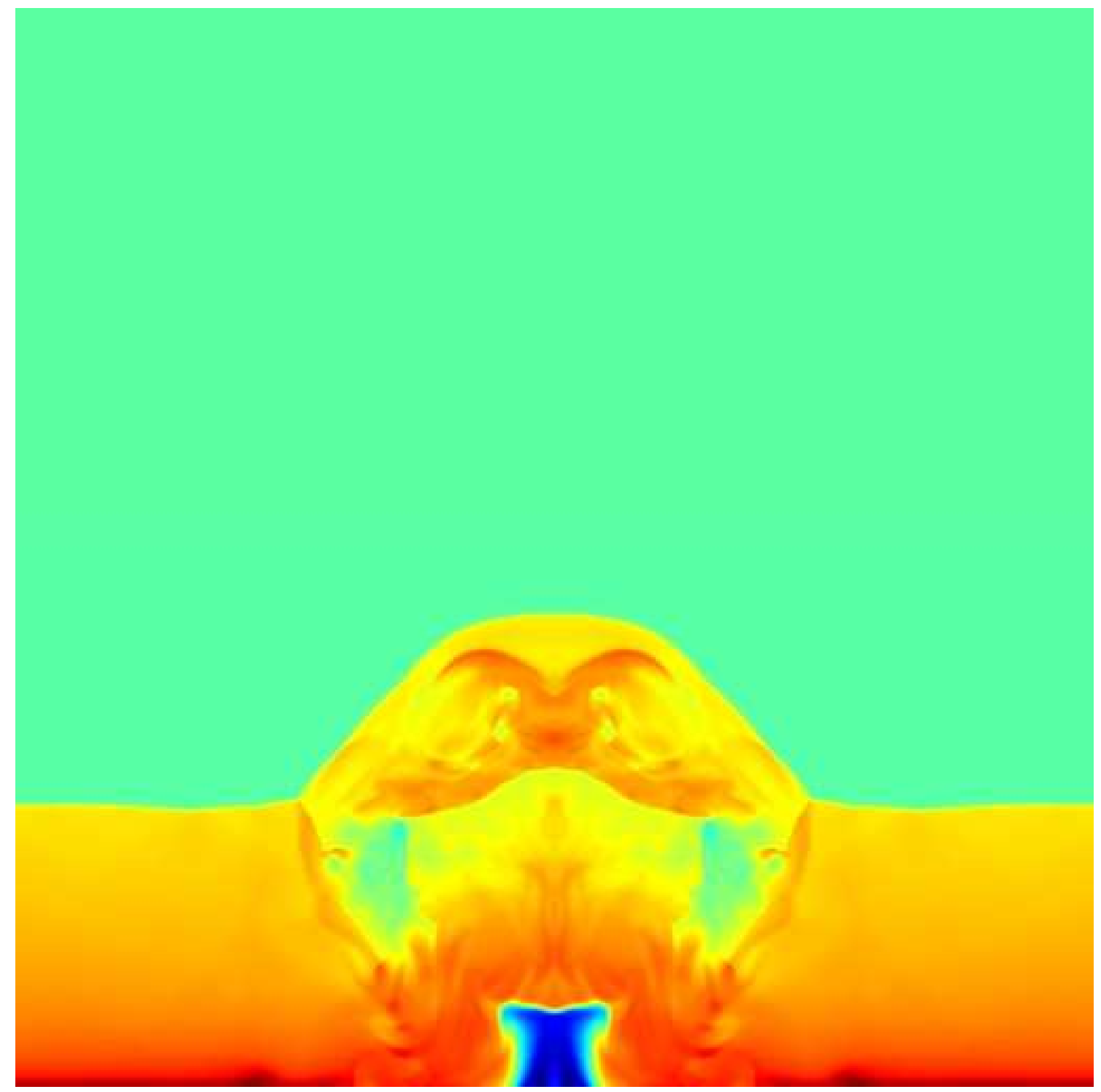

Figure 1: Figure 1 shows a relatively early time with the jet propagation roughly a third of the way into the simulation volume. The simulation shows a jet injection into a volume affected by a significant gravity gradient from the plane of the accretion disk injection with parameters $\mathrm{v} / \mathrm{c}=0.998, \mathrm{rho}_{\text {in }}=1.0$, rho $_{\text {out }}=$ 100.0 press $_{\text {in }}=5 \times 10^{-3}$, jet radius $=8 \mathrm{cells}$, simulation volume $=512^{3}$, and the scale height, agrav $=$ $-1.7 \times 10^{-4}$. A perusal of the figure shows that even at a relatively early stage of the jet evolution, the return material from the jet injection can choke off the launch process, producing an episodic injection into the ambient medium. 
between the two results. At the same time, these papers demonstrate that the ambient medium is heated and partially entrained into the jet. The analysis also shows that a relativistic, low-density jet can interpenetrate an ambient gas or plasma.

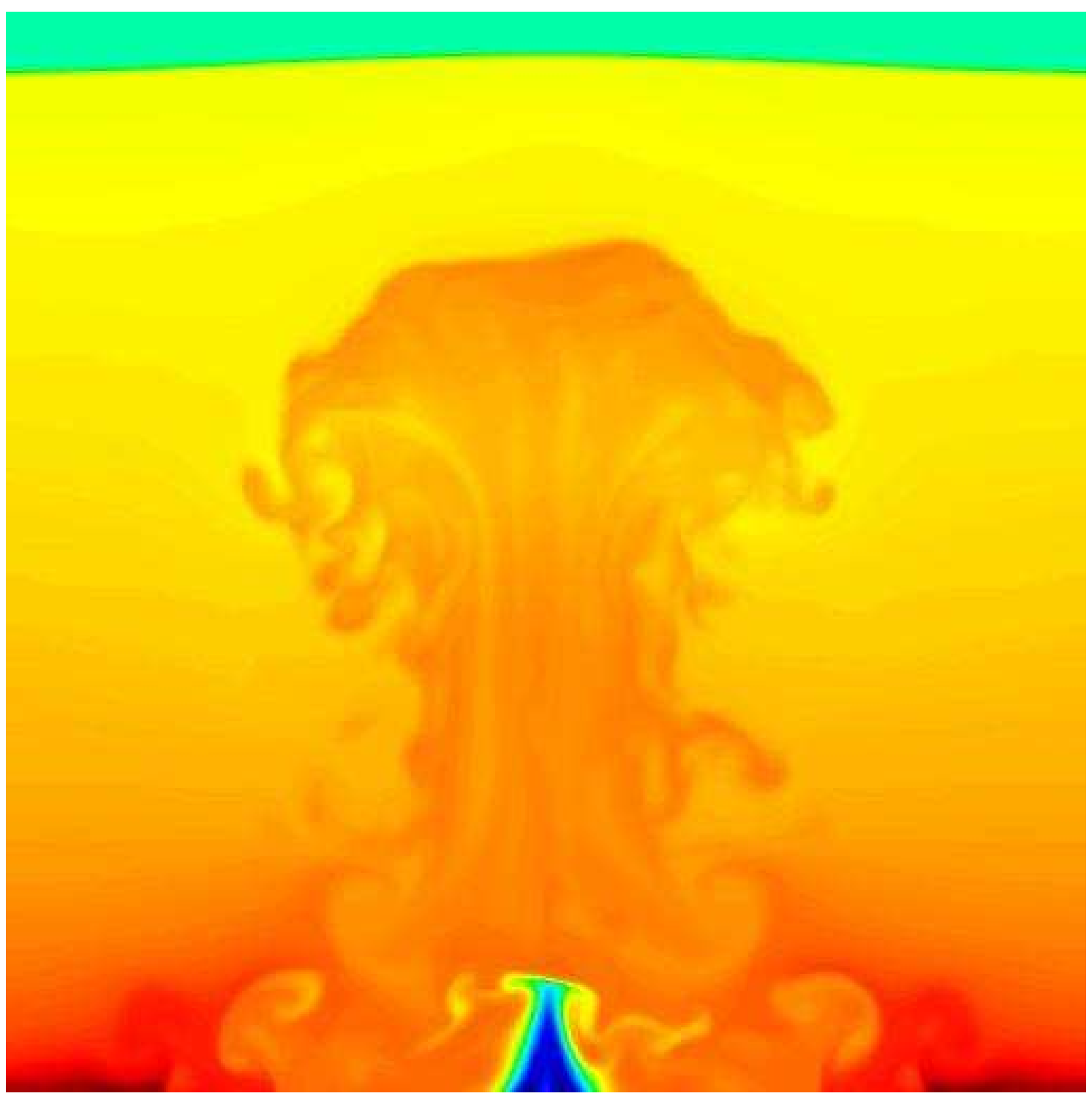

Figure 2: Figure 2 shows a relatively late time with the jet simulation in Figure 1. The simulation shows the complex structure of the late time jet evolution, wherein the injection path has been closed off by turbulent motions of the jet in its interactions with the ambient medium. propagation. The parameters of the jet are the same as those for the image in previous figure.

As part of our research into the micro-physics of the interaction of jets with an ambient medium, we continue to investigate the transfer of momentum from the jet, and expect to present these results shortly. In order to proceed to a more detailed analysis of the issue of momentum transfer, we have used modern PIC code simulations to study the dynamics of caviton formation, and have confirmed the work of Robinson and Newman (1990) in terms of the cavitons' formation, 
evolution, and collapse.

\subsection{Results of Hydrodynamical Calculations}

Figure 1 shows an $\mathrm{X}-\mathrm{Z}$ cut of a fully 3-D simulation of a relativistic jet in the hydrodynamic (RHD) regime. The figure shows jet gas density structures seen in the $\mathrm{x}-\mathrm{z}$ cross-section, a bulk velocity of $v=0.998 c$. Simulations with $512^{3}$ cells are utilized in this image. Note the well developed Rayleigh-Taylor instabilities at the jet-ambient medium boundary.

The simulation in Figure 1 was done using our modification of the PLUTO code (Mignone et al. 2007) on the NRL SGI Altix machine, POLAR. The modification provides graphical outputs as $\mathrm{X}-\mathrm{Z}$ slices at certain time steps in order to produce a convenient graphical guide. We use these images as "quick look" guides to investigate the details of the simulations. In addition, we have used the "external force" option in the code to simulate a disk gravity attraction that would illustrate coupling of the jet source to the jet-entrained ambient material. We are continuing to explore the parameter space of these jets by varying the bulk jet velocity, $\gamma$, and other jet parameters. simulation size is $\sim 5 \mathrm{kpc}$ on a side and we show the structure after $\sim 2 \times 10^{3}$ time steps. In this simulation, the jet-to-ambient medium density ratio is $1 / 100$ and the ratio of jet input pressure to ambient medium pressure is $5 \times 10^{-3}$.

Figure 2 shows the complex structure of the late-time jet evolution, wherein the injection path has been closed off by turbulent motions of the jet in its interactions with the ambient medium. The jet also has the same parameters, including $v=0.998 c$, and uses the PLUTO code with the same parameters as the jet in Figure 1. We are continuing to explore the parameter space of these jets by varying the bulk jet velocity, $\gamma$, and other jet parameters. We have used the $512^{3}$ simulation in this representation, also.

Figure 3 shows a PIC code simulation of the effects of the "microscopic" two-stream (and related) processes on the Maxwell-Boltzmann distribution of the ambient medium through which the the relativistic jet passes. The production of a high-energy tail on the thermal distribution of the gas is manifest over just a few thousand plasma cycles. Such processes can produce orders-ofmagnitude changes in the emitted line-spectrum of the gas. In the simulation shown, the ratio of the densities, $n_{b} / n_{p}=10^{-2}$.

We are continuing to explore the parameter space for the jet simulations by varying the density of the jet and the ambient medium, the jet velocity, and the relative hydrostatic pressure and the pressure of the ambient medium. For a sufficient overburden of material in the ambient medium and a low-velocity jet, it is possible to demonstrate that the jet-propagation can be thwarted. This effect might explain the relatively truncated jets associated with Seyfert galaxies.

\section{Concluding Remarks}

The effects of collective and particle processes, including plasma effects, can have observational consequences. Beall (1990) has noted that plasma processes can slow the jets rapidly, and Beall and Bednarek (1999) have shown that these effects can truncate the low-energy portion of the $\gamma$-rays spectrum (see their Figure 3).

A similar effect will occur for particle-particle productions of neutrinos, pions, and (perhaps) neutrons (Atoyan and Dermer 2003). This could also reduce the expected neutrino flux from AGN. 


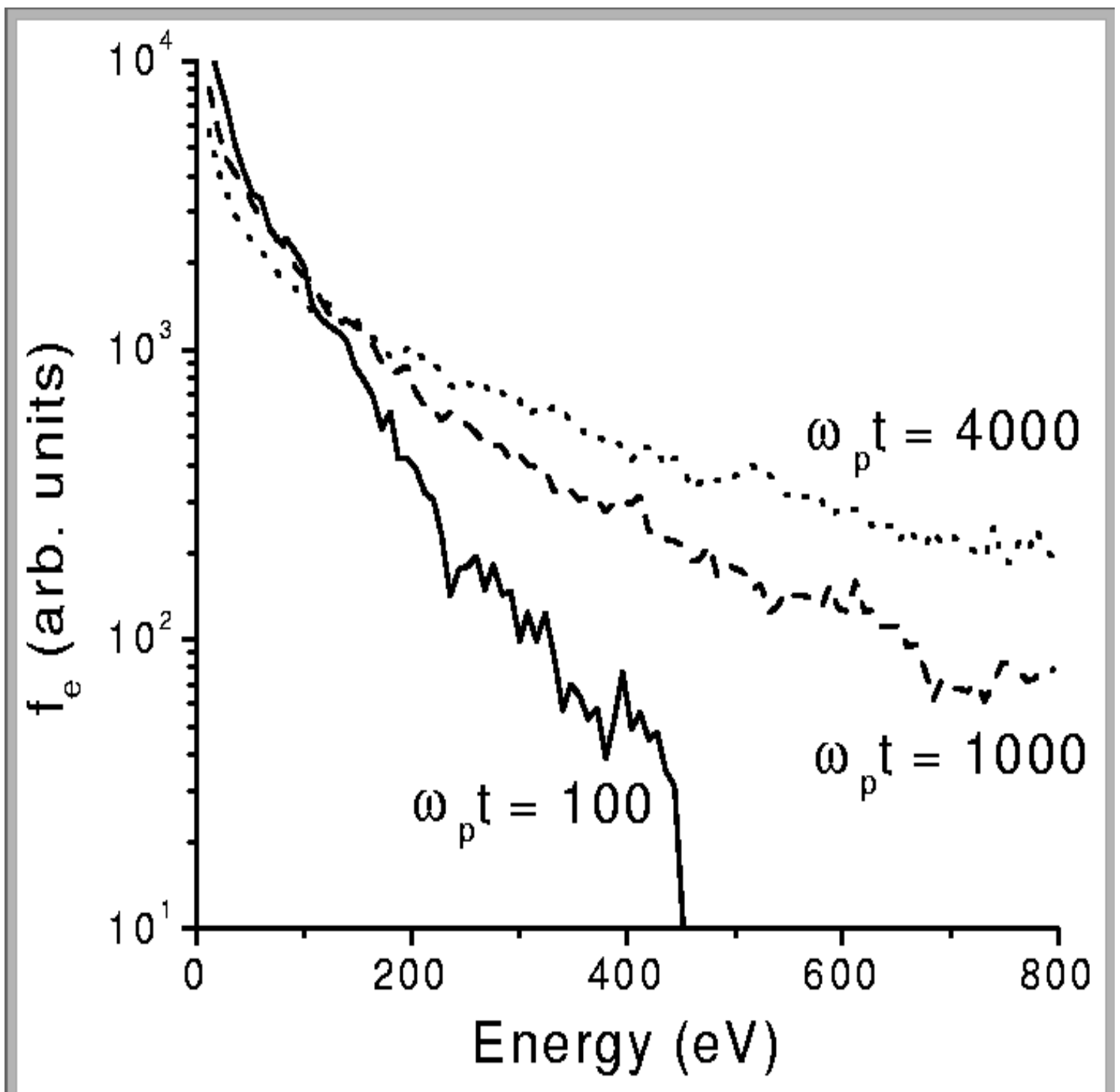

Figure 3: Figure 3 shows a PIC code simulation of the effects of the "microscopic" two-stream (and related) processes on the Maxwell-Boltzmann distribution of the ambient medium through which the relativistic jet passes. The production of a high-energy tail on the thermal distribution of the gas is manifest over just a few thousand plasma cycles. Such processes can produce orders-of-magnitude changes in the emitted linespectrum of the gas. In the simulation shown, the ratio of the densities, $n_{b} / n_{p}=10^{-2}$, where $n_{b}$ and $n_{p}$ are the jet and plasma densities, respectively. 
The presence of plasma processes in jets can also greatly enhance line radiation by generating high-energy tails on the Maxwell-Boltzmann distribution of the ambient medium, thus abrogating the assumption of thermal equilibrium.

An analytical calculation of the boost in energy of the electrons in the ambient medium to produce such a high energy tail, with $E_{\text {het }} \sim 30-100 k T$, is confirmed by PIC code simulations. Aside from altering the Landau damping rate (Rose et al. 2005), such a high-energy tail can greatly enhance line radiation over that expected for a thermal equilibrium calculation (see Beall et al. (2006), and Beall, Guillory, and Rose (1999) for a detailed discussion).

JHB and MTW gratefully acknowledge the support of the Chief of Naval Research for this project, and to the High Performance Computing Modernization Program (HPCMP), and colleagues therein, for their support in our research. Thanks also to colleagues at various institutions for their continued interest and collaboration, including Kinwah Wu, Curtis Saxton, S. Schindler and W. Kapferer, and S. Colafrancesco.

\section{References}

[1] A. M. Atoyan and C. D. Dermer, 2003, Ap. J. 586, 79.

[2] Basson, J. F., and Alexander, P., 2002, The long-term effect of radio sources on the intracluster medium, MNRAS, 339, 353.

[3] Beall, J. H., Rose, W. K., Graf, W., Price, K. M., Dent, W. A., Hobbs, R. W., Conklin, E. K., Ulrich, E. K., Dennis, B. R., Crannell, C. J., Dolan, J. F., Frost, K. J., and Orwig, E. L., 1978, Radio and X-ray variability of the nucleus of Centaurus A (NGC 5128), Ap.J., 219, 836.

[4] Beall, J. H. and Rose, W. K., 1981, On the physical environment in the nucleus of Centaurus A(NGC 5128), Ap.J., 238, 539 (also see Erratum, 1981, Ap.J., 248, 883).

[5] Beall, J. H. and Bednarek, W., 1999, On the hadronic beam model for gamma-ray production in blazars, Ap.J., 510, 188.

[6] Beall, J. H., Guillory, J., and Rose, D. V., 1999, Observational signatures in jet-cloud interactions, Mem. S. A. It., 70, 1235.

[7] Beall, J. H., Guillory, J., and Rose, D. V., 2003, AGN jet interactions with the intracluster medium, C.J.A.A., 3(Suppl.), 137.

[8] Beall, J. H., Guillory, J., Rose, D. V., Schindler, S., Colafrancesco, S., 2006, Energetics of jet interactions with the intracluster medium, C.J.A.A., 6(Suppl. 1), 283.

[9] Beall, J. H., Guillory, J., and Rose, D. V., 2010, Jet interactions with magnetized clouds, Mem. S. A. It., 81, 404.

[10] Beall J. H., 1990, Energy loss mechanisms for fast particles in an ambient medium, in Physical Processes in Hot Cosmic Plasmas, W. Brinkmann, A. C. Fabian, and F. Giobannelli, eds. (Kluwer Academic, London), p. 341.

[11] Colella, P., and Woodward, P. R., 1984, The piecewise parabolic method (PPM) for gas-dynamical simulations, J. Comp. Phys., 54, 174.

[12] Fomalont, E. B., Geldzahler, B. J., and Bradshaw, C. F., 2001, Scorpius X-1: the evolution and nature of the twin compact radio lobes, Ap.J., 558, 283. 
[13] Kaplan, S. A., and Tsytovich, V. N., 1973 Plasma Astrophysics, (Pergamon Press, Oxford).

[14] Krause, M., and Camenzind, M., 2003, Parameters for very light jets of cD galaxies, New Astron. Rev., 47, 573.

[15] Kundt, W., 1987, The sources and their models, in Astrophysical Jets and Their Engines, ed. W. Kundt (Reidel, Dordrecht, Netherlands), p. 1.

[16] Kundt, W. 1999, SS 433, Mem. S. A. It., 70, 1097.

[17] Lister, M. L., Aller, H. D., Aller, H. F., Cohen, M. H., Homan, D. C., Kadler, M., Kellermann, K. I., Kovalev, Y. Y., Ros, E., Savolainen, T., Zensus, J. A., and Vermeulen, R. C., 2009, Astronom. J., 137, 3718.

[18] Mignone, A., Bodo, G., Massaglia, S., Matsakos, T., Tesileanu, O., Zanni, C., and Ferrari, A., 2007, Ap.J. Suppl., 170, 228.

[19] Newman, D. L., Winglee, R. M., Robinson, P. A., Glanz, J., and Goldman, M. V., 1990, Simulation of the collapse and dissipation of Langmuir wave packets, Phys. Fluids B, 2, 2600.

[20] Perucho, M., 2012, Jets in high-mass microquasars, Mem. S. A. It., 83, 297.

[21] Rose, W. K., Guillory, J., Beall, J. H., and Kainer, S., 1984, The interaction of relativistic charged-particle beams with interstellar clouds, Ap.J., 280, 550.

[22] Rose, W. K., Beall, J. H., Guillory, J., and Kainer, S., 1987, Radiation from relativistic beams interacting with interstellar gas, Ap.J., 314, 95.

[23] Rose, D. V., Guillory, J. U., and Beall, J. H., 2002, Comparison of particle-in-cell simulations and a wave-population model of electron-beam-plasma interactions, Phys. Plasmas, 9, 1000.

[24] Rose, D. V., Guillory, J., and Beall, J. H., 2005, Enhanced Landau damping of finite amplitude electrostatic waves in the presence of suprathermal electron tails, Phbys. Plasmas, 12, 014501.

[25] Saxton, C. J., Bicknell, G. V., Sutherland, R. S., and Midgley S., 2005, Interactions of jets with inhomogeneous cloudy media, MNRAS, 359, 781.

[26] Scott, J. H., Holman, G. D., Ionson, J. A., and Papadopoulos, K., 1980, The heating of gas in clusters of galaxies by relativistic electrons - collective effects, Ap.J., 239, 769.

[27] Su, M., and Finkbeiner, D. P., 2012, Evidence for gamma-ray jets in the Milky Way, Ap.J., 753, 61.

[28] Zanni, C., Murante, G., Bodo, G., Massaglia, S., Rossi, P., Ferrari, A., 2005, Heating groups and clusters of galaxies: the role of AGN jets, Astron. Astrophys., 429, 399.

\section{DISCUSSION}

DIMITRI BISIKALO: How can you prove the applicability of the equations, if you know the distribution is not a Maxwellian one?

JIM BEALL: Thanks for the question. The distribution of the gas $(f(v) v x . v$. $)$ does determine the growth rate of the instabilities in the plasma wave equations. For example, a higher temperature Maxwellian tends to stabilize and dampen the growth of the instabilities, which means the twostream instability and other wave populations equilibrate at a lower level. In that case, the beam propagates further. 
In our case, we modeled the ambient medium as a two-temperature distribution, one at roughly $\mathrm{T}=10^{4} \mathrm{~K}$ and one at $\mathrm{T}=10^{5} \mathrm{~K}$, based on the PIC code simulations. That allows us to determine the growth and saturation rated of the two-stream instability waves.

GIULIO AURIEMMA: I have two questions: Which physical quantity (density, pressure,or other) is represented by the color code of your 3D graphs. Second, is any effect of the solitons visible in the simulations?

JIM BEALL: Excellent questions. The plots are only the gas density of the medium, though as a matter of course, we keep other parameters such as temperature, pressure, gas velocity, and such. In answer to your second question, if we incorporate the effects of the solitons, it reduces the propagaton length of the jet verses those without the solitons. I take your point that an extended comparison of these effects would be good.

This also bears on Dimitri's question. We would expect that the jets affected by plasma processes would typically have shorter propagation lengths. In addition, the line rations would be hard to fit with conventional, thermal models, since the ambient medium would have essentially non-thermal distibutons. 